\title{
Ocorrência de complicações crônicas e seus fatores de risco em pacientes diabéticos atendidos pelo Programa Saúde da Família de Muriaé, MG, Brasil
}

\author{
Occurrence of chronic health complications and their risk \\ factors among diabetic patients assisted by the \\ Family Health Program of Muriaé, MG, Brazil
}

Recebido em: 28/04/2015 Aceito em: 16/06/2015
Pedro Paulo Pedrosa NETTO', Marcelo Santos OLIVEIRA ${ }^{2}$, Ana Paula Rodrigues Brasil OLIVEIRA ${ }^{2}$

${ }^{1}$ Curso de Farmácia, Faculdade de Minas - FAMINAS, Av Cristiano Ferreira Varella, 555 Bairro Universitário, CEP 36880-000, Muriaé, MG, Brasil. ${ }^{2}$ Curso de Farmácia, Universidade Presidente Antônio Carlos, Campus Barbacena. Rodovia MG 338, Km 12, Colônia Rodrigo Silva, CEP 36201-143, Barbacena, MG, Brasil.E-mail: jucris78@gmail.com

\section{ABSTRACT}

The aim of this work was to analyze the occurrence of complications associated to diabetes. Also, to identify your risk factors in individuals with diabetes mellitus assisted by the Family Health Program in the city of Muriaé, MG. It was a cross-sectional quantitative study accomplished in the period of February to June of 2009 in the unit of the Family Health Program of the neighborhood São Gotardo in the city of Muriaé, MG. The sample was constituted by 50 patients. Each participant's information was obtained through a structured questionnaire. The information on the occurrence of chronic complications of diabetes has been achieved through the analysis of the patient Chart. It was observed that $23(46.0 \%)$ patients did not present any complication of the disease, and $27(54.0 \%)$ of them showed at least one health complication. The most frequent complications were retinopathy, in $18(66.67 \%)$ patients, cardiovascular diseases $(14,51.85 \%)$ and diabetic neuropathy $(11,40,74 \%)$. The risk factors more prevalent were sedentary lifestyle found in $39(78.0 \%)$ patients, central obesity in $37(74.0 \%)$ and arterial hypertension in $35(70.0 \%)$. Therefore, efforts should be done in the primary attention to the diabetic patient concerning the appropriate treatment of the disease. Because the health complications use to be associated with the diabetic patients' general health condition, reducing the quality of life of this population significantly, changes for a healthier habits should be stimulated.

Keywords: Diabetes Mellitus, chronic health complications, Public Health

\section{RESUMO}

O objetivo deste trabalho foi analisar a ocorrência de complicações associadas ao diabetes e identificar seus fatores de risco em indivíduos com diabetes mellitus assistidos pelo Programa Saúde da Família de Muriaé-MG. Foi realizado um estudo quantitativo, transversal no período de fevereiro a junho de 2009 na unidade do Programa de Saúde da Família (PSF) do bairro São Gotardo no município de Muriaé, MG. A amostra foi constituída por 50 pacientes. As informações de cada participante foram obtidas por meio de um questionário estruturado. As informações sobre a ocorrência de complicações crônicas do diabetes foram obtidas por meio da análise dos prontuários dos pacientes. Foi observado que 23 (46,0\%) pacientes não apresentavam complicação da doença, enquanto $27(54,0 \%)$ apresentavam pelo menos uma complicação, sendo as mais frequentes retinopatia, presente em $18(66,67 \%)$ pacientes, doenças cardiovasculares $(14,51,85 \%)$ e neuropatia diabética $(11,40,74 \%)$. Os fatores de risco mais prevalentes foram sedentarismo $(39,78,0 \%)$, obesidade central $(7,74,0 \%)$ e HAS $(35,70,0 \%)$. Ainda são necessários grandes esforços na atenção primária ao paciente diabético com relação ao tratamento adequado da doença e à adesão de hábitos de vida saudáveis, pois a presença de complicações associadas ao estado de saúde geral dos pacientes diabéticos diminui significativamente a qualidade de vida desta população.

Palavras chave: Diabetes Mellitus, complicações crônicas, Saúde Pública 
LISTA DE ABREVIAÇÕES: AVC- Acidente vascular cerebral; AVE- Acidente vascular encefálico; CC- Circunferência da cintura; DM- Diabetes mellitus; DCV- Doença cardiovascular; DVP- Doença vascular periférica; HAS- Hipertensão arterial sistêmica; $\mathrm{HbA}_{1 \mathrm{c}}$ - hemoglobina glicada; IM- Infarto do miocárdio; IMC- Índice de massa corporal; OMS- Organização Mundial da Saúde; PSF- Programa Saúde da Família.

\section{INTRODUÇÃO}

O Diabetes mellitus (DM) caracteriza-se como uma das principais síndromes de evolução crônica que acometem a população atual, e apresenta um significativo crescimento em sua prevalência decorrente do processo de industrialização e urbanização populacional nos últimos anos (1). Atualmente, estima-se que, no mundo, 382 milhões de pessoas são diabéticas e que este número deverá atingir 471 milhões em 2035. Cerca de $80 \%$ dos portadores de DM vivem em países em desenvolvimento, onde a epidemia tem maior intensidade (2). Segundo a Organização Mundial de Saúde (OMS), o número total de pessoas com diabetes no Brasil elevar-se-á de 4,5 milhões em 2000 para 11,3 milhões em 2030, tornando o oitavo país no mundo com o maior número de pessoas com a doença (3). A prevalência padronizada por sexo e idade, tendo como referência a população mundial, foi de $6,4 \%$ no ano de 2010. Esse número foi menor do que os estimados para os Estados Unidos da América, Canadá e México, 10,3, 9,2 e 10,8\%, respectivamente; entretanto, foi superior à prevalência estimada para o Japão (5,0\%), a Argentina (5,7\%) e o Chile (5,7\%) (4).

Esta doença representa um importante problema de saúde pública com alta morbimortalidade, com perda significativa na qualidade de vida (5). Isto se deve principalmente às complicações crônicas que dela decorrem e que frequentemente têm caráter incapacitante, comprometendo a qualidade de vida dos seus portadores e requerendo tratamento extremamente oneroso ao sistema de saúde $(5,6)$. Estimou-se que os custos dos cuidados de saúde para um indivíduo com diabetes, nos Estados Unidos, eram duas ou três vezes maiores do que para os indivíduos sem a doença (7).

O desenvolvimento de complicações crônicas no portador do Diabetes mellitus está associado à história natural da doença. As complicações são comumente classificadas como microvasculares, que resultam em nefropatia, neuropatia e retinopatia, e macrovasculares que geram as doenças cardiovasculares $(8,9,10)$. O comprometimento ateroesclerótico das artérias coronarianas, dos membros inferiores e das cerebrais é comum nos pacientes com diabetes do tipo 2, constituindo-se a principal causa de morte. Estas complicações macrovasculares podem ocorrer mesmo em estágios precoces do diabetes e se apresentam de forma mais difusa e grave do que em pessoas sem a doença (11). Os pacientes com DM do tipo 2 têm uma propensão duas a quatro vezes maior de morrer por doença cardíaca em relação aos não diabéticos, e quatro vezes mais chance de ter doença vascular periférica (DVP) e acidente vascular cerebral (AVC) (12).

As razões para a manifestação de aterosclerose acelerada em pacientes diabéticos ainda não são completamente compreendidas. Foram sugeridos como mecanismos prováveis os efeitos tóxicos diretos da glicose sobre a vasculatura, a resistência à insulina e a associação do DM a outros fatores de risco (13). O DM do tipo 2 sabidamente associa-se a vários fatores de risco cardiovasculares, incluindo hipertensão arterial sistêmica (HAS), obesidade, gordura abdominal, sedentarismo, tabagismo, alcoolismo e dislipidemia $(8,10,14)$. A duração do diabetes e seu controle também determinam o curso da macro e microangiopatia (8).

Desta maneira é de fundamental importância a identificação do perfil de risco global da população diabética e demais anormalidades da homeostase glicêmica a fim de direcionar as ações em saúde. Em vista disso o objetivo deste trabalho foi analisar a ocorrência de complicações associadas ao diabetes mellitus e identificar seus fatores de risco em indivíduos diabéticos assistidos pelo Programa Saúde da Família de Muriaé, MG.

\section{MÉTODO}

O trabalho foi delineado como um estudo quantitativo transversal, realizado no período de fevereiro a junho de 2009 na unidade do Programa Saúde da Família (PSF) do bairro São Gotardo no município de Muriaé, MG.

A amostra foi constituída por 50 pacientes com diagnóstico de Diabetes mellitus tipo 2 escolhidos por meio dos prontuários médicos, por serem os mais frequentes em consultas para o acompanhamento da doença, no ano anterior ao início da pesquisa. Dados sobre o perfil sócio-demográfico dos pacientes (idade, sexo e grau de escolaridade) e sobre hábitos de vida (tabagismo e prática de atividade física) foram obtidos por meio de entrevista, utilizando um questionário estruturado, realizada durante visita dos pesquisadores ao domicílio do paciente acompanhado por um agente de saúde. As 
informações sobre a ocorrência de complicações crônicas do diabetes (doenças cardiovasculares, retinopatia diabética, nefropatia diabética, neuropatia diabética, doenças bucais, pé diabético) foram obtidas por meio da análise das informações contidas nos prontuários dos pacientes.

Os dados de medidas antropométricas (peso e altura) e o valor da glicemia de jejum foram coletados no prontuário da última consulta médica de cada paciente.

A medida da circunferência da cintura $(\mathrm{CC})$ foi realizada pelos pesquisadores no momento da visita ao domicílio com o paciente em pé, ereto, braços soltos e situados nas laterais do corpo e abdome relaxado ao final da expiração normal, trajando roupas de pouco volume na parte superior do corpo. A CC foi aferida utilizando fita métrica inelástica, com escala de $0,5 \mathrm{~cm}$, posicionada na metade da distância entre a crista ilíaca e o rebordo costal inferior, em triplicata (15). Para a interpretação e avaliação dos resultados da $\mathrm{CC}$ foram utilizados como ponto de corte para o sexo masculino valor $>102 \mathrm{~cm} \mathrm{e}$ para o sexo feminino valor $>88 \mathrm{~cm}$ para risco de obesidade central (8).

Com os dados de altura e peso, foi determinado o Índice de Massa Corporal (IMC) para classificação do estado nutricional global segundo pontos de corte descritos na literatura (8), sendo considerado normal o indivíduo com valores de IMC entre 18,5 e $24,9 \mathrm{Kg} / \mathrm{m}^{2}$; sobrepeso, com valores entre $25,0-29,9 \mathrm{Kg} / \mathrm{m}^{2}$; obesidade classe I, com valores entre 30 e $34,9 \mathrm{Kg} / \mathrm{m}^{2}$; obesidade classe II, com valores de IMC entre 35 e $39,9 \mathrm{Kg} /$ $\mathrm{m}^{2}$ e indivíduos com obesidade classe III, com valores de $\mathrm{IMC} \geq 40 \mathrm{Kg} / \mathrm{m}^{2}$.

Para a verificação do controle glicêmico foram considerados os valores dos resultados de glicemia de jejum da última dosagem laboratorial realizada pelos pacientes. A interpretação dos resultados foi realizada com base nos valores descritos pela Sociedade Brasileira de Diabetes (16) que considera como um bom controle o valor da glicemia de jejum $<110$ $\mathrm{mg} / \mathrm{dL}$.

Foram considerados hipertensos aqueles pacientes com diagnóstico clínico prévio de Hipertensão Arterial Sistêmica (HAS) e em uso de terapia anti-hipertensiva, dados estes contidos no prontuário de cada paciente.

Foram considerados sedentários aqueles indivíduos que não atingiram a recomendação de $150 \mathrm{mi}-$ nutos por semana de atividade moderada a vigorosa, segundo o protocolo do United States Department of Health and Human Services (17), enquanto que tabagistas foram considerados os pacientes que relataram fumar diariamente.

A pesquisa seguiu as determinações da resolução 196/96 do Conselho Nacional de Saúde e foi aprovada pelo Comitê de Ética e Pesquisa da Faculdade de Minas - FAMINAS com o parecer $\mathrm{n}^{\circ}$ $001 / 2010$. Aos participantes foram explicados os objetivos da pesquisa e esclarecidos os princípios de confidencialidade, privacidade, anonimato e a garantia que a não participação ou desistência a qualquer momento da pesquisa não acarretaria prejuízos e/ou constrangimentos. Após esclarecimentos os participantes foram convidados a assinarem um Termo de Consentimento Livre e Esclarecido.

Os dados foram processados e analisados por meio dos programas Microsoft Office Excel 2003 e Graphpad prism 4 (Graphpad software, INC). A associação entre as variáveis analisadas foi verificada pelo teste de associação do Qui-quadrado $\left(\mathrm{X}^{2}\right)$ e pelo teste Exato de Fischer bicaudal. O nível de significância estabelecido foi $5 \%(\mathrm{p}<0,05)$.

\section{RESULTADOS}

$\mathrm{Na}$ população avaliada, todos os pacientes apresentavam diabetes do tipo 2. Dos 50 pacientes avaliados, 32 (64\%) eram do sexo feminino e 18 $(36 \%)$ do sexo masculino. A faixa etária variou entre 28 e 82 anos, sendo a média de idade dos pacientes 60,88 anos. A média de idade das pacientes do sexo feminino avaliadas foi 62,2 anos e dos pacientes do sexo masculino foi 58,5 anos. Em ambos os sexos, a faixa etária predominante foi de 50 a 69 anos, sendo $19(59,37 \%)$ entre as pacientes do sexo feminino e $9(50 \%)$ entre os pacientes do sexo masculino. Os pacientes apresentavam uma média de duração da doença de 10 anos, variando de 2 meses a 40 anos. Neste estudo, $96 \%$ dos pacientes relataram fazer uso de medicamentos como agentes antidiabéticos orais e/ou insulina.

A prevalência de complicações crônicas decorrentes do diabetes pode ser observada pela Figura 1. Foi observado que $23(46 \%)$ pacientes não apresentavam qualquer complicação da doença, enquanto 27 (54\%) apresentavam pelo menos uma complicação.

Entre as complicações identificadas foram encontradas alterações macrovasculares, como as doenças cardiovasculares; alterações microvasculares, como a retinopatia, nefropatia e neuropatia diabética e a presença do pé diabético e problemas bucais decorrentes da doença. 


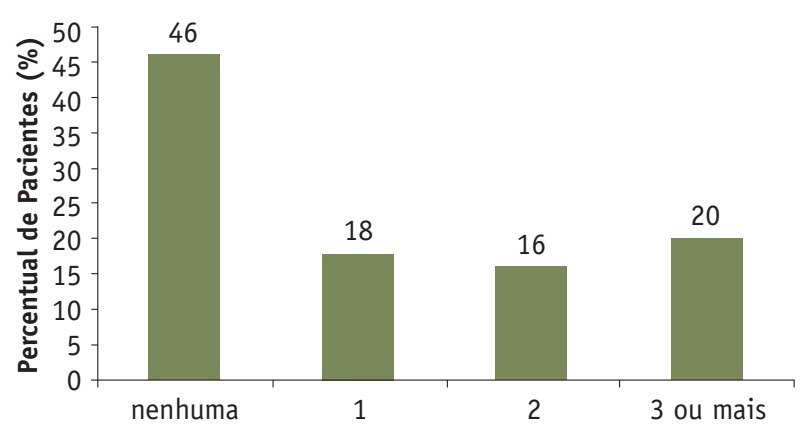

Figura 1 - Frequência de complicações crônicas do diabetes em pacientes atendidos pelo Programa Saúde da Família em Muriaé-MG

As complicações mais frequentes foram retinopatia, presente em 18 (66,67\%) pacientes; doenças cardiovasculares encontradas em 14 (51,85\%) pacientes, e neuropatia diabética presente em $11(40,74 \%)$ pacientes (Figura 2).

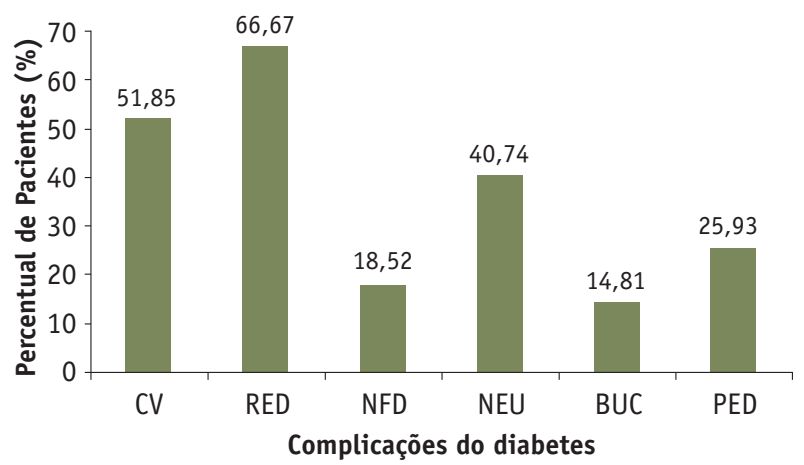

Figura 2 - Prevalência das complicações crônicas do diabetes mellitus tipo 2 entre pacientes atendidos no Programa Saúde da Família em Muriaé, MG. CV = doenças cardiovasculares, RED = retinopatia diabética, NFD $=$ nefropatia diabética, NEU = neuropatia diabética, $B U C=$ doenças bucais, $P E D=$ pé diabético

Ao analisar a Tabela 1 não foi observada diferença com relação à frequência de complicações crônicas do diabetes e o sexo dos pacientes, apenas uma proporção maior de mulheres $(56,2 \%)$ em relação aos homens (50\%). Com relação à idade e o aparecimento de complicações crônicas da doença não foi observada nenhuma diferença na frequência de complicações nas diferentes faixas etárias. Contudo, a maior frequência de complicações do diabetes $(71,4 \%)$, ocorreu entre os pacientes situados na faixa etária de 50 a 59 anos (Tabela 1).

Ao analisar a relação entre grau de escolaridade do paciente e a ocorrência de complicações foi observa- da uma diferença significativa $(\mathrm{p}<0,05)$, sendo que dos 8 pacientes que tiveram menos de 4 anos de estudo, 7 (87,5\%) já possuíam complicações crônicas do diabetes, percentual esse bem superior em relação aos pacientes que tiveram 4 a 8 anos e mais de 8 anos de estudo, 55,6\% e $33,3 \%$, respectivamente (Tabela 1 ).

O controle glicêmico foi verificado a partir do resultado da última dosagem de glicemia em jejum dos pacientes. Foi admitido como um bom controle, conforme recomendações da Sociedade Brasileira de Diabetes (16) glicemia de jejum $<110 \mathrm{mg} / \mathrm{dL}$, sendo este resultado encontrado em apenas $10(20 \%)$ pacientes. No entanto, não houve diferença estatística $(\mathrm{p}>0,05)$ na frequência de complicações crônicas do diabetes entre pacientes que estavam com a glicemia controlada ou não, sendo verificada a presença de complicações em $4(40,0 \%)$ pacientes com glicemia de jejum inferior a $110 \mathrm{mg} / \mathrm{dL}$ e em 23 (57,5\%) pacientes com glicemia $\geq 110 \mathrm{mg} / \mathrm{dL}$.

Com relação aos fatores de risco para desenvolvimento das complicações micro e macrovasculares foi pesquisada a presença de obesidade, hipertensão arterial sistêmica (HAS), tabagismo, sedentarismo e obesidade central (medida através da circunferência da cintura). Os fatores de risco mais prevalentes foram sedentarismo, encontrado em 39 (78,0\%) pacientes, obesidade central em 37 (74,0\%) pacientes e HAS em 35 (70,0\%) pacientes. A obesidade e o tabagismo foram encontrados em $15(30,0 \%)$ e $5(10,0 \%)$ pacientes, respectivamente. A análise da relação entre a presença dos fatores de risco e a presença de complicações crônicas do diabetes não demonstrou diferença significativa para obesidade, tabagismo, sedentarismo e obesidade central. Porém a associação entre hipertensão e ocorrência de complicações crônicas foi significativa $(\mathrm{p}<0,05)$.

Conforme mostrado na Figura 3, apenas 4 (8\%) pacientes não apresentaram fatores de risco, sendo que nenhum destes apresentou complicações crônicas da doença. Além disso, todos os pacientes que apresentaram complicações possuíam pelo menos um fator de risco.

\section{DISCUSSÃO}

O diabetes mellitus tipo 2 é a forma presente em 90\%-95\% dos casos de diabetes e caracteriza-se por defeitos na ação e na secreção da insulina. 
Tabela 1 - Perfil sócio-demofgráfico e condições de saúdede pacientes diabéticos atendidos no Programa Saúde da Família em Muriaé, MG

\begin{tabular}{|c|c|c|c|c|c|c|c|}
\hline \multirow[t]{2}{*}{ Variáveis } & \multicolumn{2}{|c|}{ Total } & \multicolumn{4}{|c|}{ Complicações do diabetes } & \multirow[b]{2}{*}{ p* } \\
\hline & $\mathrm{N}$ & $\%$ & Sim & $\%$ & Não & $\%$ & \\
\hline Sexo & & & & & & & $0,67^{a}$ \\
\hline Masculino & 18 & 36,0 & 9 & 50,0 & 9 & 50,0 & \\
\hline Feminino & 32 & 64,0 & 18 & 56,2 & 14 & 43,8 & \\
\hline
\end{tabular}

\begin{tabular}{|c|c|c|c|c|c|c|c|}
\hline Faixa etária & & & & & & & $0,22^{\mathrm{a}}$ \\
\hline$<40$ anos & 4 & 8,0 & 1 & 25,0 & 3 & 75,0 & \\
\hline 40 a 49 anos & 5 & 10,0 & 1 & 20,0 & 4 & 80,0 & \\
\hline 50 a 59 anos & 14 & 28,0 & 10 & 71,4 & 4 & 28,6 & \\
\hline 60 a 69 anos & 14 & 28,0 & 7 & 50,0 & 7 & 50,0 & \\
\hline$>70$ anos & 13 & 26,0 & 8 & 61,5 & 5 & 38,5 & \\
\hline
\end{tabular}

\begin{tabular}{|l|c|c|c|c|c|c|}
\hline Anos de estudo & & & & & $0,04^{\mathrm{a}}$ \\
\hline$\leq 4$ anos & 8 & 16,0 & 7 & 87,5 & 1 & 12,5 \\
\hline 4 a 8 anos & 27 & 54,0 & 15 & 55,6 & 12 & 44,4 \\
\hline$>8$ anos & 15 & 30,0 & 5 & 33,3 & 10 & 66,7 \\
\hline
\end{tabular}

\begin{tabular}{|c|c|c|c|c|c|c|c|}
\hline Glicemia $<110 \mathrm{mg} / \mathrm{dL}$ & & & & & & & $0,48^{b}$ \\
\hline Sim & 10 & 20,0 & 4 & 40,0 & 6 & 60,0 & \\
\hline Não & 40 & 80,0 & 23 & 57,5 & 17 & 42,5 & \\
\hline
\end{tabular}

\begin{tabular}{|c|c|c|c|c|c|c|c|}
\hline Obesidade (IMC $\geq$ 30) & & & & $0,76^{\text {b }}$ \\
\hline Sim & 15 & 30,0 & 9 & 60,0 & 6 & 40,0 \\
\hline Não & 35 & 70,0 & 18 & 51,4 & 17 & 48,6 \\
\hline
\end{tabular}

\begin{tabular}{|l|l|l|l|l|l|l|l|}
\hline Hipertensão & & & & & $0,02^{\text {b }}$ \\
\hline Sim & 35 & 70,0 & 23 & 65,7 & 12 & 34,3 & \\
\hline Não & 15 & 30,0 & 4 & 26,7 & 11 & 73,3 \\
\hline
\end{tabular}

\begin{tabular}{|c|c|c|c|c|c|c|c|}
\hline Tabagismo & & & & & & \\
\hline Sim & 5 & 10,0 & 4 & 80,0 & 1 & 20,0 & \\
\hline Não & 45 & 90,0 & 23 & 51,1 & 22 & 48,9 & \\
\hline
\end{tabular}

\begin{tabular}{|l|c|c|c|c|c|c|c|c|}
\hline Sedentarismo & \multicolumn{3}{|c|}{} & & & $0,08^{\mathrm{b}}$ \\
\hline Sim & 39 & 78,0 & 24 & 61,5 & 15 & 38,5 \\
\hline Não & 11 & 22,0 & 3 & 27,3 & 8 & 72,7 \\
\hline \\
\hline Obesidade central & & & & & & \\
\hline Sim & 37 & 74,0 & 22 & 59,5 & 15 & 40,5 \\
\hline Não & 13 & 26,0 & 5 & 38,5 & 8 & 61,5 \\
\hline
\end{tabular}

${ }^{*} p<0,05$ considerado significativo; $a=$ Teste Qui-quadrado; $b=$ Teste Exato de Fischer bicaudal

Em geral ambos os defeitos estão presentes quando a hiperglicemia se manifesta, porém pode haver predomínio de um deles. Pode ocorrer em qualquer idade, mas é geralmente diagnosticado após os 40 anos. Os pacientes não são dependentes de insulina exógena para sobrevivência, porém podem necessitar de tratamento com insulina para a obtenção de um controle metabólico adequado (18). Neste trabalho a maioria dos pacientes diabéticos apresentou idade entre 50 e 59 anos, com tempo médio da doença de 10 anos sendo o tratamento medicamentoso feito com antidiabéticos orais e/ou insulina. 
A história natural do diabetes é marcada pelo aparecimento de complicações crônicas classificadas como micro e macrovasculares. Estas respondem por expressiva morbimortalidade, com taxas de mortalidade cardiovascular e renal, cegueira, amputação de membros e perdas de função e qualidade de vida muito superior a indivíduos sem diabetes (8).

No presente trabalho a maioria dos pacientes diabéticos (54\%) apresentou pelo menos uma complicação crônica da doença registrada em prontuário. Vieira Santos et al. (19) encontraram 58,9\% de pacientes com pelo menos uma complicação do diabetes.

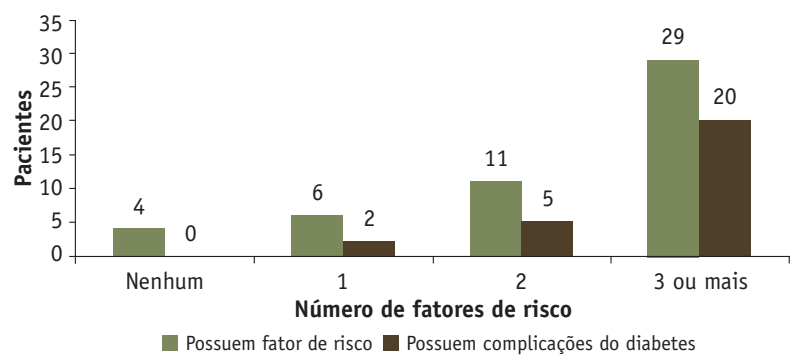

Figura 3 - Número de fatores de risco entre pacientes com complicações crônicas do diabetes assistidos pelo Programa Saúde da Família em Muriaé, MG.

Entre os pacientes avaliados a complicação mais frequente foi a retinopatia, seguida pelas doenças cardiovasculares e a neuropatia. A retinopatia diabética acomete cerca de $40 \%$ dos pacientes diabéticos e é a principal causa de cegueira em pacientes entre 25 e 74 anos (20). Esta pode ser evitada através de medidas adequadas, que incluem, além do controle da glicemia e da pressão arterial, a realização do diagnóstico em uma fase inicial e passível de intervenção (21). É também um indicador precoce de início das complicações microvasculares e do risco de comprometimento renal (8).

As doenças cardiovasculares associadas ao diabetes incluem a doença arterial coronariana, a doença cerebrovascular (Acidente Vascular Encefálico - AVE) e vascular periférica, sendo estas as complicações que apresentam maior morbimortalidade, pois entre 50 a $80 \%$ da mortalidade na população diabética pode ser atribuída a estas complicações $(8,14)$.

Além de incapacitação e morte prematura pelas complicações decorrentes de aterosclerose e trombose vascular, a doença cardiovascular (DCV) no paciente diabético cursa com hospitalização mais prolongada (18). A prevenção e o tratamento das doenças cardiovasculares em pacientes com diabetes seguem noções semelhantes ao das pessoas sem diabetes. Intervenções que controlem os fatores de risco para complicações têm seu benefício comprovado na prevenção destas complicações (8).
Outra complicação crônica muito comum do diabetes é a neuropatia diabética, caracterizada pela degeneração progressiva dos axônios das fibras nervosas e que compreende um conjunto de síndromes clínicas que comprometem o sistema nervoso periférico sensitivo, motor e autonômico, de modo isolado ou difuso, nos segmentos proximal ou distal, de instalação aguda ou crônica, de caráter reversível ou irreversível, que se manifesta de forma silenciosa ou com fortes quadros sintomáticos $(5,8)$.

Embora no presente trabalho não tenha sido encontrada significância estatística entre o sexo e a ocorrência de complicações crônicas, a literatura tem apontado para a variação na prevalência da doença entre os sexos, no tempo e no espaço $(18,22,23)$. A maior proporção de mulheres acometidas por complicações decorrentes do diabetes no presente estudo pode ser explicada pelo fato destas pacientes procurarem mais frequentemente os serviços de saúde (24). Outro fato a ser considerado é que no Brasil, como em outras sociedades contemporâneas, a expectativa de vida é sempre mais elevada para as mulheres do que para os homens. Consequentemente, aumentam as chances das mulheres apresentarem um percentual maior de complicações que os homens (19).

Diferentemente de Vieira Santos et al. (2008) houve diferença significativa entre a educação formal e a ocorrência de complicações do diabetes (19). O grau de instrução dos pacientes favorece o controle e manejo adequado do diabetes, e consequentemente, reflete a ocorrência de possíveis complicações crônicas da doença. Os pacientes que não possuem instrução ou possuem apenas o ensino fundamental, apresentam dificuldades no acesso às informações, como também na compreensão dos mecanismos complexos do diabetes e de seu tratamento, reduzindo tanto as oportunidades de aprendizagem quanto os cuidados com a saúde (25).

O controle metabólico adequado associado a medidas preventivas e curativas previnem ou retardam o aparecimento das complicações crônicas do diabetes, resultando em melhor qualidade de vida ao indivíduo diabético (26). A hiperglicemia é um dos fatores envolvidos na etiologia das complicações crônicas do Diabetes mellitus. Acredita-se que as mais importantes complicações do diabetes como retinopatia, nefropatia e neuropatia, sejam devidas à hiperglicemia (27). Ainda que para complicações microvasculares o controle glicêmico tenha maior significância, os fatores de risco são semelhantes aos expostos para doença macrovascular $(5,8)$.

Na prática clínica, a avaliação do controle glicêmico é feita através da utilização de dois recursos laboratoriais: os testes de glicemia e os testes de hemoglobina glicosilada $\left(\mathrm{A}_{1} \mathrm{C}\right)$, cada um com seu significado clínico 
específico e ambos considerados como recursos complementares para a correta avaliação do estado de controle glicêmico em pacientes diabéticos (16).

O controle glicêmico dos pacientes avaliados, analisado através do resultado do último exame laboratorial de glicemia de jejum, não foi satisfatório, pois apenas $20 \%$ apresentaram glicemia de jejum $<110 \mathrm{mg} / \mathrm{dL}$, assim como verificado por Scheffel et al. 2004 , cujo percentual de pacientes diabéticos com glicemia $<110 \mathrm{mg} /$ $\mathrm{dL}$ foi de apenas $16 \%$ (28). Quanto à relação entre controle glicêmico e ocorrência de complicações, os resultados aqui apresentados são semelhantes aos encontrados por Vieira Santos et al. (2008). A falta de significância estatística entre glicemia alterada e ocorrência de complicações pode ser devido à determinação da glicemia em amostra isolada (19). Os testes de glicemia refletem o nível glicêmico atual e instantâneo no momento exato do teste, enquanto os testes de hemoglobina $\mathrm{A}_{1} \mathrm{C}$ refletem a glicemia média pregressa dos últimos dois a quatro meses, sendo este marcador mais confiável do controle glicêmico no paciente diabético (16).

Embora os mecanismos que resultem no aparecimento das complicações do diabetes ainda não estejam inteiramente esclarecidos, a duração da doença e seu controle associados a fatores de risco, como hipertensão arterial, obesidade, gordura abdominal, sedentarismo, tabagismo, alcoolismo e dislipidemia, determinam o curso da micro e macroangiopatia (8). As DCV são uma causa importante de óbito nos EUA, e o diabetes ocupa lugar de destaque, visto que em cerca de $25 \%$ dos pacientes diabéticos a primeira manifestação é o infarto de miocárdio (IM) ou a morte súbita (29). Quando ocorre o primeiro infarto, a sobrevida nos indivíduos diabéticos é bem menor. $\mathrm{O}$ diabetes confere um alto risco para novo evento coronariano, dentro de dez anos, em razão da frequente associação com os múltiplos fatores de risco cardiovasculares (18).

No presente trabalho foi verificada a presença de múltiplos fatores de risco nos pacientes avaliados, sendo o sedentarismo, a obesidade central e a hipertensão arterial, os mais prevalentes. A presença de fatores de risco nos pacientes estudados também foi importante e considerável em outros estudos $(11,28,30)$. Schaan et al. (2004) e Scheffel et al. (2004) verificaram percentuais semelhantes ao presente trabalho para a presença de obesidade nos pacientes diabéticos estudados sendo, $35,3 \%$ e $36,0 \%$, respectivamente $(28,31)$. A HAS tam- bém foi prevalente em mais de $70 \%$ dos pacientes avaliados (28).

O sedentarismo tem sido considerado fator de risco para a mortalidade prematura. Estima-se que anualmente a falta de atividade física seja responsável por $10 \%$ a $16 \%$ dos casos de diabetes e $22 \%$ das doenças isquêmicas (19). É indicada a prática de atividade física de forma regular a todos os pacientes portadores de diabetes, pois, gera melhoria no controle metabólico, reduz a necessidade de hipoglicemiantes, nos pacientes com sobrepeso ou obesos ajuda a promover o emagrecimento, diminui os riscos de doença cardiovascular e melhora a qualidade de vida (32).

Estudos demonstraram que a adiposidade abdominal aumentada em indivíduos diabéticos, independente da adiposidade global, também se associa à mortalidade por doenças cardiovasculares, além de contribuir para pior controle metabólico da doença (33).

A hipertensão arterial afeta $50 \%$ da população diabética comparada com $20 \%$ da população geral sendo um importante facilitador para o desenvolvimento de retinopatia, nefropatia e doença cardiovascular aumentando drasticamente o risco de morbidade e mortalidade cardiovascular (34). No presente trabalho, foi encontrada uma relação significativa entre a presença de hipertensão nos pacientes e a ocorrência de complicações crônicas da doença.

Além disso, foi verificado que os pacientes diabéticos que não possuíam nenhum fator de risco, não apresentaram nenhuma complicação crônica da doença, enquanto que aqueles pacientes que apresentavam pelo menos um fator de risco possuíam complicações.

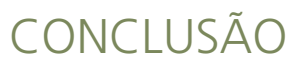

A presença de complicações crônicas decorrentes do diabetes e do seu mau controle verificados neste trabalho, por meio do resultado da última dosagem de glicemia de jejum do paciente, além da presença de fatores de risco para o seu desenvolvimento, mostra que ainda são necessários grandes esforços na atenção primária ao paciente diabético com relação ao tratamento adequado da doença e à adesão de hábitos de vida saudáveis. Isso se faz necessário, pois a presença de complicações associadas ao estado de saúde geral dos pacientes diabéticos diminui significativamente a qualidade de vida desta população. 
1. Souza LJ, Chalita FEB, Reis AFF, Teixeira CL, Gicovate Neto C, Bastos DA, Souto Filho JTD, Souza TF, Côrtes VA. Prevalência de diabetes mellitus e fatores de risco em Campos dos Goytacazes, RJ. Arq Bras Endocrinol Metabol. 2003; 47:69-74. DOI: 10.1590/S000427302003000100011 .

2. IDF. International Diabetes Federation. Diabetes Atlas. Belgium: IDF. 2013.

3. Rathmann W, Giani G. Global prevalence of diabetes: estimates for the year 2000 and projections for 2030 . Diabetes Care. 2004; 27(10):1047-1053. DOI: $10.2337 /$ diacare.27.10.2568.

4. Shaw JE, Sicree RA, Zimmet PZ. Global estimates of the prevalence of diabetes for 2010 and 2030. Diabetes Res Clin Pr. 2010; 87(1): 4-14. DOI: 10.1016/j.diabres.2009.10.007.

5. BRASIL. Ministério da Saúde. Plano de reorganização da atenção à hipertensão arterial e ao Diabetes mellitus: hipertensão arterial e diabetes mellitus. Brasília (DF); 2001.

6. Selli L, Papaléo LK, Meneghel SN, Tornero JZ. Técnicas educacionales en el tratamiento de la diabetes. Cad Saúde Pública. 2005; 21(5): 1366-72. DOI: 10.1590/S0102$311 X 2005000500008$.

7. Bahia LR, Araujo DV, Schaan BD, Dib SA, Negrato CA, Leão MPS et al. The costs of type 2 diabetes mellitus outpatient care in the Brazilian Public Health System. Value in Health. 2011; 14(S):37-40. DOI: 10.1016/j. jval.2011.05.009.

8. BRASIL. Ministério da Saúde. Secretaria de Atenção à Saúde. Departamento de Atenção Básica. Diabetes mellitus. Cadernos de Atenção Básica. Brasília (DF); 2006.

9. Lisbôa HRK, Souilljee M, Cruz CS, Zoletti L, Gobbato DO. Prevalência de hiperglicemia não diagnosticada nos pacientes internados nos hospitais de Passo Fundo, RS. Arq Bras Endocrinol Metabol. 2000; 44: 220-226. DOI: 10.1590/S0004-27302000000300006.

10. SBD. Sociedade Brasileira de Diabetes. Consenso Brasileiro sobre diabetes 2002: diagnóstico e classificação do diabetes melito e tratamento do diabetes melito tipo 2. Rio de Janeiro: Diagraphic; 2003.

11. ADA. American Diabetes Association. Diabetes complications. In: Diabetes 2001 vital statistics. Virginia: Port City Press, Inc; 2001. p. 43- 74.

12. Kanters SD, Banga JD, Stolk RP, Algra A. Incidence and determinants of mortality and cardiovascular events in diabetes mellitus: a meta-analysis. Vasc Med. 1999; 4: 67-75. DOI: $10.1177 / 1358836$ X9900400203.

13. Haffner SM, Lehto S, Ronnemma T, Pyorala K, Laakso M. Mortality from coronary heart disease in subjects with type 2 diabetes and in nondiabetic subjects with and without prior myocardial infarction. N Engl J Med. 1998; 339:229-34. DOI: 10.1056/NEJM199807233390404.
14. Monteiro AG, Rosário F, Torre JB. Complicações Cardiovasculares na diabetes Prevenções Primária e Secundária nos CSP. Rev Port Clin Geral. 2007; 23:627647.

15. SBC. Sociedade Brasileira de Cardiologia. IV Diretriz Brasileira Sobre Dislipidemias e Prevenção da Aterosclerose Departamento de Aterosclerose da Sociedade Brasileira de Cardiologia. Arq Bras Cardiol. 2007; 88 (1): 2-19.

16. SBD. Sociedade Brasileira de Diabetes. Diretrizes da Sociedade Brasileira de Diabetes. Rio de Janeiro: Diagraphic; 2008.

17. USDHHS. Physical Activity Guidelines Advisory Committee Report 2008. Washington: United States Department of Health and Human Services; 2008.

18. SBD. Sociedade Brasileira de Diabetes. Tratamento e acompanhamento do Diabetes mellitus. Diretrizes da Sociedade Brasileira de Diabetes. Rio de Janeiro: Diagraphic; 2007.

19. Vieira Santos ICR, Carvalho EF, Souza WV, Medeiros MCWC, Nóbrega MGL, Lima PMS. Complicações crônicas dos diabéticos tipo 2 atendidos nas Unidades de Saúde da Família, Recife, Pernambuco, Brasil. Rev Bras Saúde Matern Infant. 2008; 8 (4): 427-433. DOI: 10.1590/ S1519-38292008000400008.

20. Aiello LP, Gardner TW, King GL, Blankenship G, Cavallerano JD, Ferris FL, Klein R. Diabetic retinopathy. Technical review. Diabetes Care.1998; 21:143-156.

21. Gross JL, Nehme M. Detecção e tratamento das complicações crônicas do diabetes melito: Consenso da Sociedade Brasileira de Diabetes e Conselho Brasileiro de Oftalmologia. Rev Ass Med Brasil. 1999; 45(3): 279-284. DOI: 10.1590/S0104-42301999000300014.

22. Franco LJ, Mameri C, Pagliaro H, Iochida LC, Goldenberg P. Diabetes como causa básica ou associada de morte no Estado de São Paulo, Brasil, 1992. Rev Saúde Pública. 1998; 32: 237-245. DOI: 10.1590/S003489101998000300006.

23. Freitas MTA, Jorge MHPM. Diabetes mellitus na população idosa em município da Região Sul do Brasil: um estudo da mortalidade e morbidade hospitalar. Arq Bras Endocrinol Metab. 2004; 48: 505-512. DOI: 10.1590/ S0004-27302004000400011.

24. Miranzi SSC, Ferreira FS, Iwamoto HH, Pereira GA, Miranzi MAS. Qualidade de vida de indivíduos com Diabetes mellitus e hipertensão acompanhados por uma equipe de saúde da família. Texto Contexto Enferm. 2008; 17(4): 672-679. DOI: 10.1590/S0104-07072008000400007.

25. Rocha RM, Zanetti ML, Santos MA. Comportamento e conhecimento: fundamentos para prevenção do pé diabético. Acta Paul Enferm. 2009; 22(1): 17-23. DOI: 10.1590/S0103-21002009000100003. 
26. Paiva DCP, Bersusa AAS, Escuder MML. Avaliação da assistência ao paciente com diabetes e/ou hipertensão pelo Programa Saúde da Família do Município de Francisco Morato, São Paulo, Brasil. Cad Saúde Pública. 2006; 22(2): 377-85. DOI: 10.1590/S0102$311 X 2006000200015$.

27. Corrêa ZMS, Eagle R. Aspectos patológicos da retinopatia diabética. Arq Bras de Oftalmol. 2005. 68: 410-414. DOI: $10.1590 /$ S0004-27492005000300028.

28. Scheffel RS et al. Prevalência de complicações micro e macrovasculares e de seus fatores de risco em pacientes com diabetes melito do tipo 2 em atendimento ambulatorial. Rev Assoc Med Bras. 2004. 50: 263-267. DOI: 10.1590/S0104-42302004000300031.

29. Mazzone T. O papel da tomografia computadorizada por feixe de elétrons para mensuração da aterosclerose da artéria coronária. Current Diabetes Reports - Latin America. 2004; 3: 230-236.
30. Ferreira CLRA, Ferreira MG. Características epidemiológicas de pacientes diabéticos da rede pública de saúde - análise a partir do sistema HiperDia. Arq Bras Endocrinol Metab. 2009; 53: 80-86. DOI: 10.1590/ S0004-27302009000100012.

31. Schaan BA, Harzheim E, Gus I. Perfil de risco cardíaco no diabetes mellitus e na glicemia de jejum alterada. Rev Saúde Pública. 2004; 38(4): 529-536. DOI: 10.1590/ S0034-89102004000400008.

32. MINAS GERAIS. Secretaria de Estado de Saúde. Atenção a saúde do adulto: hipertensão e diabetes. Saúde em Casa. Belo Horizonte (MG); 2006.

33. Van Gaal L, Rillaerts E, Creten W, De Leew I. Relationship of body fat distribution pattern to atherogenic risck factors in NIDDM: preliminary results. Diabetes Care. 1988; 11(2):103-106. DOI: 10.2337/diacare.11.2.103.

34. Freire RD. Recomendações nutricionais no diabetes me1litus. O Mundo da Saúde. 2001; 25: 168-184.

134 